\title{
The crystal structure of a Polerovirus exoribonuclease- resistant RNA shows how diverse sequences are integrated into a conserved fold
}

\author{
ANNA-LENA STECKELBERG, ${ }^{1,4}$ QUENTIN VICENS, ${ }^{1,4}$ DAVID A. COSTANTINO, ${ }^{1}$ JAY C. NIX, ${ }^{2}$ \\ and JEFFREY S. KIEFT ${ }^{1,3}$ \\ ${ }^{1}$ Department of Biochemistry and Molecular Genetics, University of Colorado Denver School of Medicine, Aurora, Colorado 80045, USA \\ ${ }^{2}$ Molecular Biology Consortium, Advanced Light Source, Lawrence Berkeley National Laboratory, Berkeley, California 94720, USA \\ ${ }^{3}$ RNA BioScience Initiative, University of Colorado Denver School of Medicine, Aurora, Colorado 80045, USA
}

\begin{abstract}
Exoribonuclease-resistant RNAs (xrRNAs) are discrete elements that block the progression of $5^{\prime}$ to $3^{\prime}$ exoribonucleases using specifically folded RNA structures. A recently discovered class of xrRNA is widespread in several genera of plant-infecting viruses, within both noncoding and protein-coding subgenomic RNAs. The structure of one such xrRNA from a dianthovirus revealed three-dimensional details of the resistant fold but did not answer all questions regarding the conservation and diversity of this xrRNA class. Here, we present the crystal structure of a representative polerovirus xrRNA that contains sequence elements that diverge from the previously solved structure. This new structure rationalizes previously unexplained sequence conservation patterns and shows interactions not present in the first structure. Together, the structures of these xrRNAs from dianthovirus and polerovirus genera support the idea that these plant virus xrRNAs fold through a defined pathway that includes a programmed intermediate conformation. This work deepens our knowledge of the structure-function relationship of xrRNAs and shows how evolution can craft similar RNA folds from divergent sequences.
\end{abstract}

Keywords: RNA structure; viral RNA; exoribonuclease resistance; RNA pseudoknot; X-ray crystallography

\section{INTRODUCTION}

RNA structure-dependent exoribonuclease resistance is now well-established as a means of subgenomic RNA production or maintenance by many viruses (Slonchak and Khromykh 2018). This mechanism depends on discrete, specifically folded RNA elements in viral genomes called xrRNAs (for "exoribonuclease-resistant RNAs"). xrRNAs block the $5^{\prime}$ to $3^{\prime}$ progression of processive cellular exoribonucleases using only a folded RNA element, without accessory proteins (Chapman et al. 2014a). In so doing, they protect downstream RNA from degradation. xrRNAs were discovered in flaviviruses and have since been identified in other viruses, including plant viruses of the Luteoviridae and Tombusviridae families (Iwakawa et al. 2008; Pijlman et al. 2008; Steckelberg et al. 2018a). In flaviviruses, xrRNAs have been found exclusively at the beginning of the $3^{\prime}$ untranslated region (UTR) of the viral genome where

\footnotetext{
${ }^{4}$ Joint first authors.

Corresponding author: Jeffrey.Kieft@cuanschutz.edu

Article is online at http://www.rnajournal.org/cgi/doi/10.1261/rna. 076224.120.
}

they function in the generation of viral noncoding RNAs, whereas in Luteoviridae and Tombusviridae, xrRNAs are associated with both noncoding and protein-coding regions of the viral genome (Fig. 1A; Steckelberg et al. 2018b). Despite this evidence that xrRNAs are widespread we are only beginning to understand the principles of xrRNA folding that lead to structures capable of blocking host cell exoribonucleases.

Crystal structures of xrRNAs from flaviviruses revealed that two pseudoknots and other long-range interactions create a ring-like fold that wraps around the $5^{\prime}$ end of the xrRNA (Chapman et al. 2014a; Akiyama et al. 2016; Jones et al. 2020). Mechanistic studies suggest that this ring protects downstream RNA by "bracing" against the surface of the exoribonuclease, acting as a physical block to the $5^{\prime}$ to $3^{\prime}$ progression of the enzyme (Chapman et al. 2014a;

C) 2020 Steckelberg et al. This article is distributed exclusively by the RNA Society for the first 12 months after the full-issue publication date (see http://rnajournal.cshlp.org/site/misc/terms.xhtml). After 12 months, it is available under a Creative Commons License (Attribution-NonCommercial 4.0 International), as described at http:// creativecommons.org/licenses/by-nc/4.0/. 
A

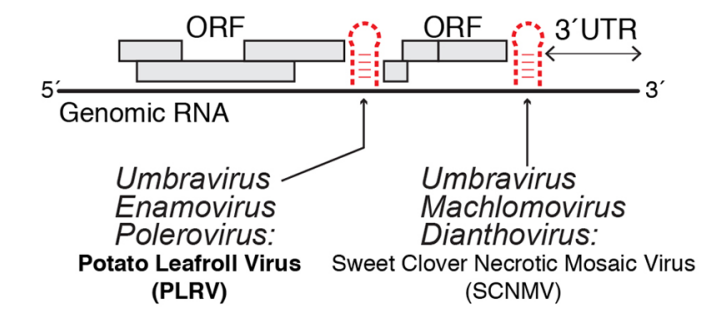

B

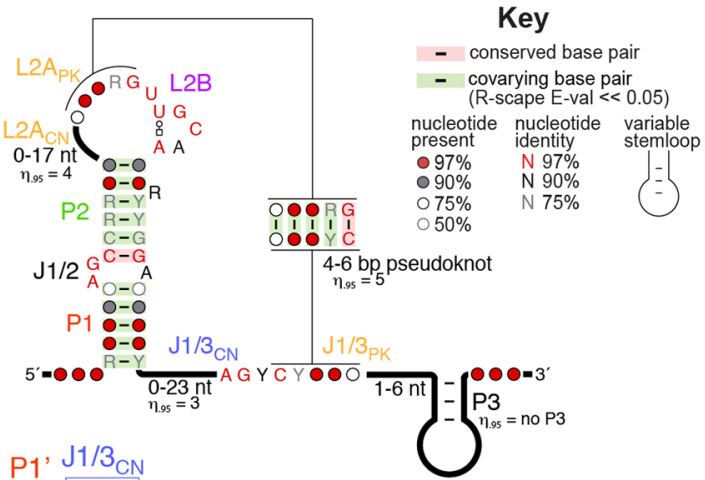

C

PLRV
MYDV-RMV
SABYV
SLPV
BrYV
PDMV
PeWBVYV
UPOIV1
BWYV
PABYV
OV5
ZABYV
WLYaV
SCNMV
RCNMV
MCMV
OPMV
ZPE1
2D

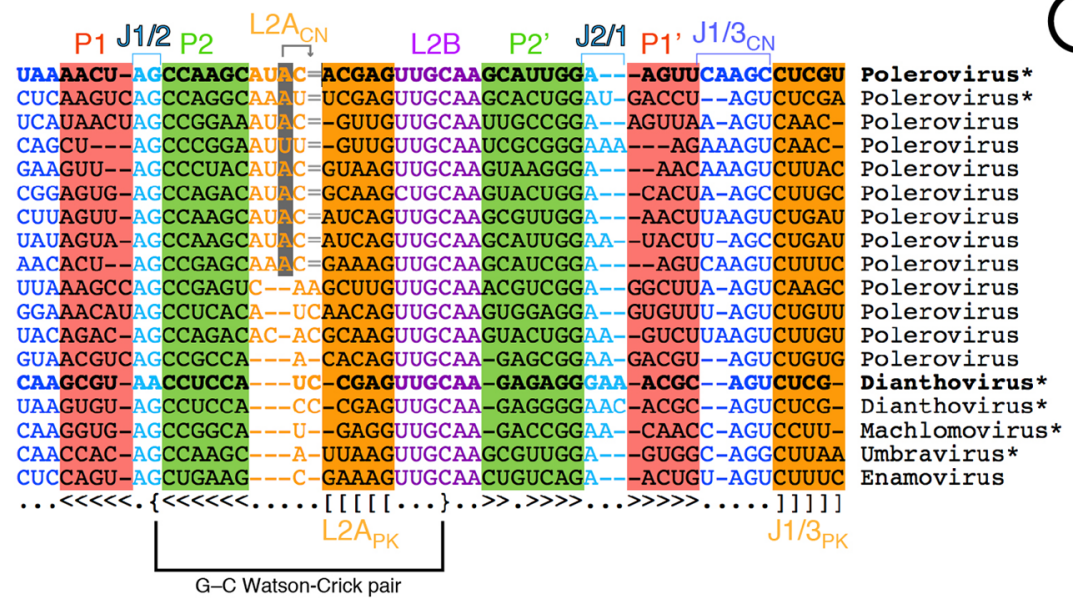

FIGURE 1. A brief overview of plant xrRNAs. (A) Depending on the plant virus family, xrRNAs are located within intergenic regions and/or at the beginning of the $3^{\prime}$ UTR. (B) Consensus secondary structure for $x r R N A_{L T} S$. The 95 th percentile $\left(\eta_{.95}\right)$ is indicated for all variable regions. Covariation analysis was expanded and updated from Steckelberg et al. (2018b). (C) Alignment of xrRNA $L T$ sequences from select plant viruses. Bold sequences (PLRV and SCNMV) indicate a crystal structure is available; ${ }^{*}$, xrRNA confirmed to be resistant to Xrn1 in vitro; gray highlight in L2A $\mathrm{A}_{C N}$; the gray shaded nucleotide aligns $2 \mathrm{nt}$ downstream in three dimensions, as indicated by the arrow pointing to the "=" sign. The G-C pair involving J1/2 and L2B is labeled. The complete alignment is shown in Supplemental Figure S1.

Akiyama et al. 2016; MacFadden et al. 2018). A recent crystal structure of an xrRNA from sweet clover necrotic mosaic virus ([SCNMV]; a dianthovirus from the Tombusviridae family) showed a different three-dimensional fold conferring exoribonuclease resistance (Steckelberg et al. 2018a). Specifically, the SCNMV xrRNA was captured in a folding intermediate state that contains a Watson-Crick base-paired helix (P1) that must be unwound to allow the formation of a pseudoknotted ring structure. Single-molecule Förster resonance energy transfer (smFRET) experiments revealed that the SCNMV xrRNA's intermediate state can be remodeled by the helicase activity of the arriving exoribonuclease to favor the resistant pseudoknotted fold (Steckelberg et al. 2018a). While a ring encircling the $5^{\prime}$ end seems to be thus far a defining characteristic of all xrRNAs, the degradation-induced structural remodeling seen in SCNMV's xrRNA appears to be unique. Overall, the $x r R N A s$ of the flavivirus-type $\left(x r R N A_{F}\right)$ and the dianthovirus-type ( $\left.x r R N A_{D}\right)$ are distinguishable based on their different underlying three-dimensional folds.

The discovery of intramolecular interactions within xrRNA $A_{D}$ that could not be predicted from the sequence informed subsequent bioinformatic-based approaches that identified $>40$ new examples of this class of xrRNA (Steckelberg et al. 2018b). These xrRNAs pervade the Luteoviridae and Tombusviridae families in general, and the Umbravirus and Polerovirus genera in particular (polerovirus sequences make for $\sim 2 / 3$ of this alignment). Some of the deadliest viruses in agriculture contain an xrRNA, such as potato leafroll virus ([PLRV], leading responsible virus for worldwide potato yield loss [Wale et al. 2008]), maize chlorotic mottle virus ([MCMV], responsible for $90 \%$ maize/corn yield loss in sub-Saharan Africa [Mahuku et al. 2015]), and maize yellow dwarf virus-RMV ([MYDVRMV], formerly BYDV-RMV [Krueger et al. 2013], responsible for $30 \%$ yield loss in affected winter wheat fields [Perry et al. 2000]). All newly identified xrRNAs in Luteoviridae are found in intergenic regions, whereas xrRNAs from Tombusviridae are generally found at the beginning of the $3^{\prime}$ UTR (Steckelberg et al. 2018b). Because they are widespread in the Luteoviridae and Tombusviridae, we now refer to this class as $\operatorname{xrRNA}_{L T}$ in this manuscript rather than $x r R N A_{D}$. Interestingly, the many newly discovered $x_{R R N A} A_{L T}$ contain conserved sequence elements absent from the crystallized SCNMV xrRNA (Fig. 1B,C; Steckelberg et al. 2018b). This raised the questions of whether 
the newly identified $x r R N A_{L T S}$ form the same fold as the dianthovirus xrRNA and, if so, how they accommodate these different sequence elements.

To address the universality of the interactions necessary to support an $x r R N A_{L T}$ fold as first seen in SCNMV, we explored the structure and function of the $\operatorname{xrRNA} A_{L T}$ from PLRV using $X$-ray crystallography and in vitro assays of exoribonuclease resistance. In the crystal, the PLRV xrRNA $A_{L T}$ adopts a folding intermediate conformation similar to that of the SCNMV xrRNA $A_{L T}$. But as the PLRV xrRNA $A_{L T}$ is $\sim 10 \%$ longer than the SCNMV xrRNA $A_{L T}$, our structure reveals how extra nucleotides leading to extended helices and loops generate interactions and motifs not seen in the SCNMV structure. Using site-directed mutagenesis coupled with functional assays, we show how these interactions are critical for exoribonuclease resistance. Together, our results rationalize sequence conservation patterns of $\operatorname{xrRNA}_{L T}$, thereby providing insights into the mechanism of programmed exoribonuclease resistance in the viral world.

\section{RESULTS AND DISCUSSION}

\section{Crystallization of a polerovirus xrRNA $_{\text {LT }}$}

The previously reported structure of the SCNMV xrRNA $A_{L T}$ revealed how an RNA sequence of only $44 \mathrm{nt}$ adopts a specific fold to generate an exoribonuclease-resistant structure (Steckelberg et al. 2018a). Because the dianthovirus sequences in the original $x_{R R N A}$ LT alignment were not representative of the broader diversity in the expanded alignment (Fig. 1C; Supplemental Fig. S1), the SCNMV structure could not fully explain the consensus secondary structure (Fig. 1B). In particular, all $x r R N A_{L T S}$ contain a guanosine in the J1/2 internal loop between the P1 and P2 stems (G8 in PLRV; loop previously referred to as L1; Figs. $1 C$ and 2A), except for SCNMV, which contains an adenosine. In SCNMV, the Hoogsteen side of $A 8$ interacts with the sugar edge of $\mathrm{G} 33$ in $\mathrm{J} 2 / 1$, an interaction that could not be reproduced by swapping the $A$ and the $G$ as in the majority of the sequences in the alignment. Additionally, $x r R N A_{L T}$ sequences are longer for poleroviruses than for dianthoviruses, with extra nucleotides in $\mathrm{P} 2$ and in the less-conserved regions within the apical loop L2A (including the part involved in the pseudoknot) and the adjoining region $\mathrm{J} 1 / 3$ (Fig. 1B,C). Examining the SCNMV structure did not suggest how these extra nucleotides could be accommodated. Finally, the SCNMV $x r R N A_{L T}$ was crystallized in a state that appeared to be a necessary folding intermediate. Additional structural information was needed to confirm this as an authentic intermediate or to visualize the final pseudoknotted state.

To allow a structural comparison with the SCNMV

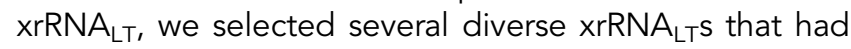
the conserved $\mathrm{G}$, expanded regions in P2, L2A, and J1/3, and various pseudoknot lengths (4-5 bp). Of these, the $\operatorname{xrRNA}_{L T}$ from PLRV yielded crystals that diffracted to 5-7 Å on our home source but that we could not reproduce (construct \#1, Supplemental Fig. S2A). Diffraction was
A

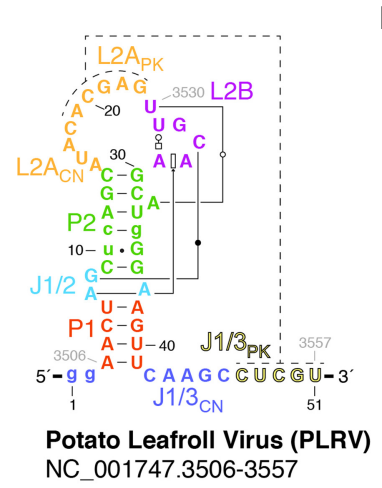

C

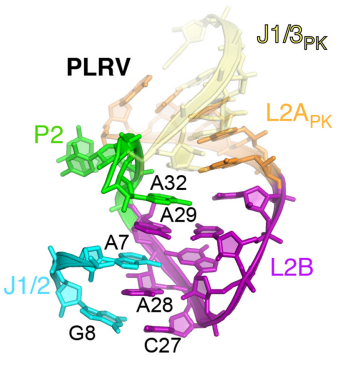

D

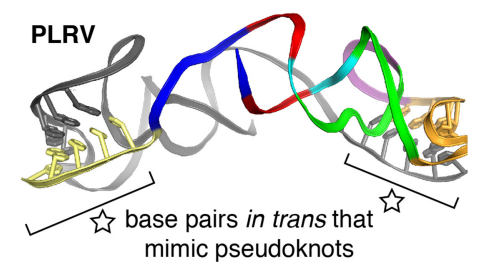

B

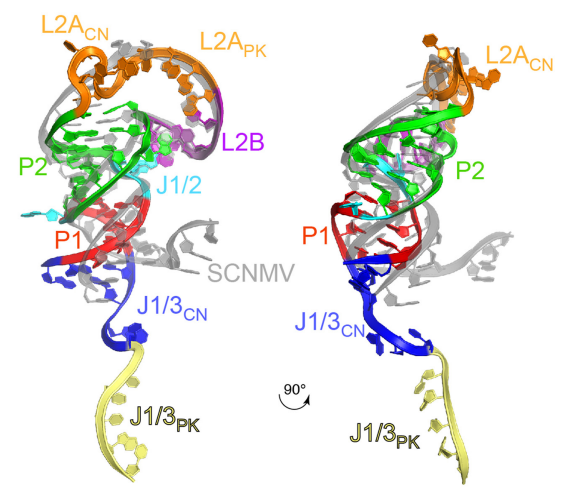

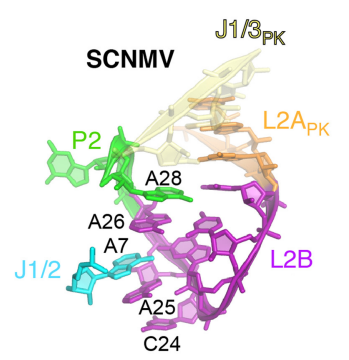

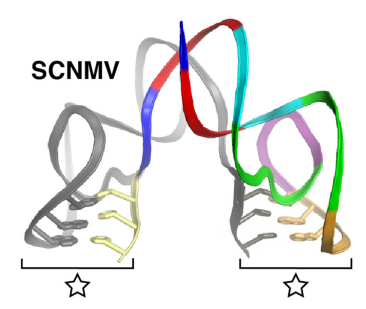

FIGURE 2. Structure of the PLRV xrRNA. (A) Secondary structure of the crystallized PLRV RNA, with matching colors to Figure 1B,C. Engineered nucleotides (see Materials and Methods) are shown in lower case. Non-Watson-Crick base pairs are according to the Leontis-Westhof annotation (Leontis and Westhof 2001). (B) Ribbon representation of the PLRV xrRNA $A_{L T}$ structure in colors to match (A), over that from SCNMV in gray (two orthogonal views). (C) Side-by-side comparison of the conserved structural core involving J1/2, L2B, and P2. (D) The PLRV and SCNMV xrRNA $A_{L T S}$ both crystallize as a domain-swap dimer, in which the intramolecular pseudoknot is replaced by intermolecular base-pairing (indicated by a star). 
improved to $\sim 3 \AA$ Apon adding of $100 \mathrm{mM}$ guanidinium hydrochloride during crystallization (see Materials and Methods) and introducing a G.U wobble base pair "phasing module" at various locations to aid with phasing using iridium(III) hexammine (Supplemental Fig. S2A; Keel et al. 2007). Introducing the phasing module in constructs \#1-4 did not alter the ability of the RNA to resist exoribonuclease-catalyzed degradation (Supplemental Fig. S2B). The structure of construct \#3 was eventually solved to 2.6 A from combined data sets collected at a synchrotron using single anomalous dispersion phasing at the L-I edge of iridium (Supplemental Table S1; Supplemental Fig. S3; Batey and Kieft 2016).

\section{A common intermediate fold for $\operatorname{xrRNA}_{L T}$ elements}

The global structure of the PLRV $x r R N A_{L T}$ is similar in shape and architecture to that of the SCNMV $x$ rRNA $A_{L T}$. Like SCNMV, the PLRV xrRNA $A_{L T}$ folds as a "bent-over" stemloop in which $L 2 B$ creates a docking point for long-range tertiary interactions involving nucleotides located in $\mathrm{J} 1 / 2$ (Fig. 2A,B). The all-atom root-mean square deviation (RMSD) is $0.9 \AA$ for a superimposition of $L 2 A_{P K}$ (the section of L2A involved in pseudoknot formation; to be distinguished from $L 2 A_{C N}$, the $5^{\prime}$ part of $L 2 A$ connecting $P 2$ to L2A $A_{P K}$ ) and L2B from SCNMV and PLRV, indicating that the two RNAs share a similar "core" structure (Fig. 2C). The improvement in diffraction quality upon addition of guanidinium is likely due to binding of four guanidinium molecules around $\mathrm{J} 1 / 2$ and $\mathrm{J} 2 / 1$, at sites where iridium (III) hexammine complexes bind in the SCNMV structure (Supplemental Fig. S4A). Three of the guanidinium molecules stack to form a spine in the deep groove of three consecutive guanines (G34-G36, Supplemental Fig. S4A,B). Stacking is a known property of guanidinium ( $\mathrm{Di}$ Tondo and Pritchard 2012; Vazdar et al. 2018), but it had not been observed to that extent within a complex with RNA. Guanidiniums interact with the RNA in a manner reminiscent of that seen in guanidinium riboswitches (Breaker et al. 2017; Nelson et al. 2017); compare, for example, guanidinium bound to G36-G39 in PLRV to the guanidinium binding site within a guanidine-II riboswitch (Supplemental Fig. S4C; Reiss and Strobel 2017). While guanidinium stacking improved crystal diffraction, it did not alter the biochemical behavior of the RNA. Specifically, the addition of low concentrations of guanidine hydrochloride did not change the equilibrium between the monomeric and a dimeric state of the RNA in native gel electrophoresis (Supplemental Fig. S5A,B), nor did it impair Xrn1 resistance in in vitro RNA degradation assays (Supplemental Fig. S5C).

As in the SCNMV xrRNA $A_{L T}$, the PLRV xrRNA $A_{L T}$ was crystallized in a "domain-swap dimer" conformation, in which the intramolecular pseudoknot required for activity was replaced by interactions in trans between the two molecules in the asymmetric unit (Supplemental Fig. S3A-C). Five Watson-Crick base pairs formed between the L2APK region of one molecule and $\mathrm{J} 1 / 3_{\mathrm{PK}}$ of the second molecule (Fig. 2D). Hence, the PLRV RNA was captured in a similar folded state to the SCNMV RNA (Steckelberg et al. 2018a), although the relative angle between the two molecules is about three times wider for PLRV (Fig. 2D). Because the P1 stem formed, but not the pseudoknot, in both SCNMV and PLRV xrRNA $A_{L T}$, this "open" state is likely an authentic intermediate conformation adopted by diverse $\operatorname{xrRNA}_{L T S}$ to ensure that the correct topology is ultimately achieved (Supplemental Fig. S3C; Steckelberg et al. 2018a). This conclusion is further supported by previous smFRET and functional studies that showed that mutations favoring this intermediate state are not deleterious to exoribonuclease resistance, while those destabilizing this state reduce exoribonuclease resistance (Steckelberg et al. 2018a). Taking into account the sequence and secondary structure conservation of known $\operatorname{xrRNA}_{L T S}$ (Fig. 1B,C; Supplemental Fig. S1), we can expect that every xrRNA $A_{L T}$ generally follows a similar folding strategy, in which the initial formation of $\mathrm{P} 1$ positions the $3^{\prime}$ end relative to the $5^{\prime}$ end of the RNA, and thereby ensures that adoption of the final "closed" pseudoknotted form creates a protective ring encircling the $5^{\prime}$ end (Supplemental Fig. $\mathrm{S} 3 \mathrm{C}$ ). As previously proposed, this closed form, not yet directly visualized, would be promoted by the helicase activity of the approaching exoribonuclease, which unwinds P1 (Steckelberg et al. 2018a).

\section{An unexpected $\mathrm{G}-\mathrm{C}$ base pair contributes to the "bent-over" conformation}

The PLRV and SCNMV xrRNA architectures are globally similar (Supplemental Fig. S6); however, several local structural differences account for the diversity in xrRNA $A_{L T}$ sequences. Although A8 is paired with G34 in SCNMV, the $>97 \%$ conserved $\mathrm{G} 8$ and $\mathrm{A} 37$ at the equivalent positions in PLRV and all other $x r R N A_{L T}$ s identified are both bulged out from the stem (Fig. 3A). A37 forms crystal contacts (Supplemental Fig. S3D), but G8 pairs with C27 in L2B (equivalent to C24 in SCNMV; Figs. $2 \mathrm{C}$ and $3 \mathrm{~A}$ ). The G8-C27 pair caps a stack of purines from $L 2 B, J 1 / 2$, and $\mathrm{P} 2$, which is otherwise conserved between SCNMV and PLRV (Fig. 2C). Because the overall conformation of L2B relative to $\mathrm{P} 1$ and $\mathrm{P} 2$ is similar between the two structures, this additional base pair could be viewed as stabilizing but not altering the $\operatorname{xrRNA}_{L T}$ conformation (Figs. $2 \mathrm{C}$ and $3 \mathrm{~A}$ ). The local changes at J1/2 and L2B seen in the PLRV structure may seem minimal, but they contribute to explaining the $>97 \%$ sequence conservation at $\mathrm{J} 1 / 2$ and L2B in a manner that the SCNMV structure could not.

The high conservation of the G8-C27 pair suggests it is important for exoribonuclease resistance. To test this, we generated two mutant RNAs that each disrupted the ability 
A

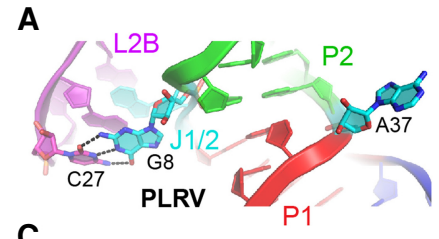

C

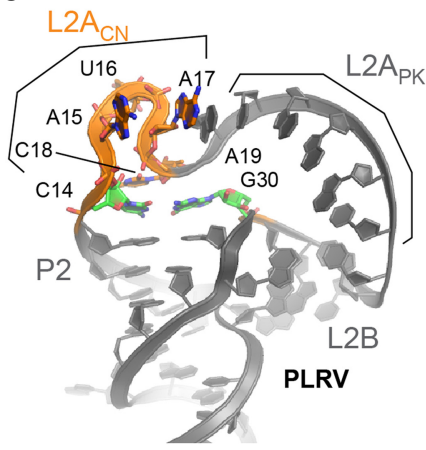

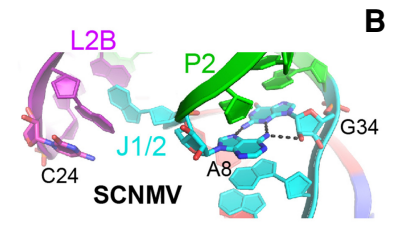

B

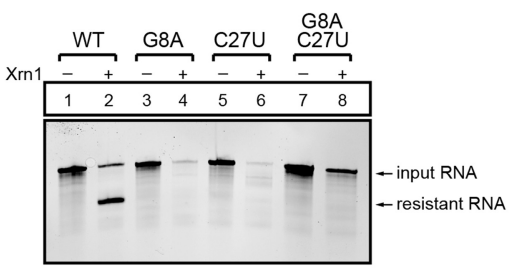

D

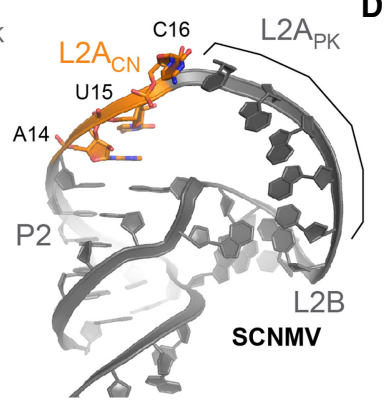

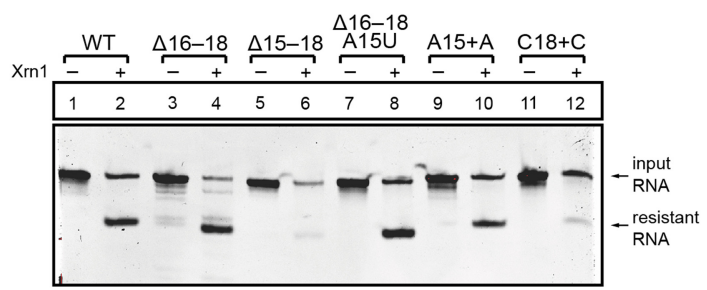

FIGURE 3. Structural diversity of the $x r R N A_{L T}$ fold. (A) Sequence differences at J1/2 lead to the formation of the G8-C27 pair with L2B in PLRV (left; color coding as in Fig. 1C). Similar view for SCNMV (right). (B) In vitro Xrn1 degradation assay of PLRV xrRNA (C) Architecture of the L2A $A_{C N}$ and L2A $A_{P K}$ regions in PLRV (left) and SCNMV (right). (D) In vitro Xrn1 degradation assay of PLRV xrRNA WT and L2ACN mutants.

of these two bases to form a Watson-Crick base pair: G8A and C27U (Supplemental Fig. S7). When challenged with the recombinant $5^{\prime}$ to $3^{\prime}$ exoribonuclease $\mathrm{Xrn} 1$ in our established exoribonuclease resistance assay (Chapman et al. 2014b), both mutant RNAs were degraded, indicating they are unable to block progression of the nuclease (Fig. 3B, lanes 3-6). A double-mutant G8A + C27U was designed to restore the ability of the Watson-Crick pair to form (Supplemental Fig. S7). Yet, this mutant was also unable to block progression of the exoribonuclease (Fig. 3B, lanes 7 and 8), suggesting that the specific identity and configuration of the base pair is important. This result is consistent with the sequence alignment, which shows no covariation at these positions (Supplemental Fig. S1). Substitution at these positions likely results in local structural changes that prevent formation of this tertiary base pair. Hence, the conservation of G8 and C27 serves not only to create a base pair, but also to promote structures that make these two bases available to pair. The fact that this pair is not present in SCNMV suggests it may not be required in all contexts, as evolution can craft compensatory stabilizing interactions.

\section{PLRV's longer sequence is accommodated within the active fold}

The P2 stem and the predicted pseudoknot are longer by one base pair in PLRV compared to SCNMV. L2A $\mathrm{A}_{\mathrm{CN}}$ is also longer (by one nucleotide) in PLRV. Finally, the J1/3 connector $\left(\mathrm{J} 1 / 3_{\mathrm{CN}}\right)$ is longer by two nucleotides in PLRV (Fig. 1C; next section). The PLRV xrRNA structure shows how these additional nucleotides are accommodated. In the
SCNMV structure, L2A $A_{C N}$ has a 5'-AUC-3' sequence, while PLRV has 5'-AUAC-3', which leads to a significant structural adjustment. For SCNMV, A14 and U15 stack against P2, and $\mathrm{C} 16$ stacks against the pseudoknot-like structure formed in trans. For PLRV, the equivalent A15 and U16 flip out in opposite directions, and C18, equivalent to C16 from SCNMV, stacks against the elongated P2 (Fig. 3C; Supplemental Fig. S3D). Spatially, C14 from the C-G pair extending $\mathrm{P} 2$ and $\mathrm{C} 18$ in PLRV are in similar locations to A14 and U15 in SCNMV. The protrusion comprising A15-A17 in PLRV is accommodated by stacking of A15 and $A 17$ against L2A $A_{P K}$ (via A19; Fig. $3 C$ ) and $A 37$ from a symmetry-related molecule (Supplemental Fig. S3D). $\mathrm{U} 16$ is in the vicinity of another symmetry-related molecule, although the poor quality of the map for that base suggests it is dynamic (Supplemental Fig. S3D). This side-by-side comparison of the $\operatorname{xrRNA}_{L T}$ structures from SCNMV and PLRV reveals extensive differences that were not apparent from sequence alignments but that show how differences in the sequences are accommodated in a globally similar fold.

To test whether the alternative stacking strategy against $L_{2} A_{P K}$ involving a distorted backbone of $L 2 A_{C N}$ is required for resistance, we tested xrRNA mutants that alter L2A $A_{C N}$ (Supplemental Fig. S7). Removal of nucleotides 16-18 in PLRV to return to an SCNMV-like $L_{2} A_{C N}$ does not affect resistance, though additionally removing A15 does (Fig. 3D, lanes 3-6). Most likely, removal of A15 would make L2A $A_{C N}$ too short to support the proper conformation of $L 2 A_{P K}$ and L2B. Replacing A15 with a $U$ in the 16-18 deletion mutant does not affect resistance (Fig. 3D, lanes 7 and 8), suggesting that the presence of a nucleotide at that position is 
more important than its identity. Notably, xrRNA $A_{L T}$ tolerates the presence of an extra nucleotide in L2A $A_{P K}$ after A15, but not after C18 (Fig. 3D, lanes 9-12). A straightforward interpretation of these results is that stacking of $\mathrm{A} 17$ and $\mathrm{C} 18$ is important to support the active fold, while stacking of A15 may only occur due to the presence of A37 from a symmetry-related molecule. Overall, this analysis helps to make sense of the generally longer $\mathrm{L}_{2} \mathrm{~A}_{\mathrm{CN}}$ and $\mathrm{P} 2$ stems in poleroviruses compared to dianthoviruses (Supplemental Fig. S1).

\section{Conserved interactions in the domain-swap dimer have a functional role}

PLRV and SCNMV xrRNA $A_{L T}$ both crystallized in open conformations in which the pseudoknot is replaced by similar base pairs in trans ("domainswapped dimer state"; Fig. 2D; Supplemental Fig. S3C). The intermolecular dimer contacts likely created favorable crystal packing, and dimerization is favored at high RNA concentrations used for crystallization. Using electrophoretic mobility shift assays (EMSAs), we showed that at the RNA concentration of $150 \mu \mathrm{M}$ used for crystallization, around $50 \%$ of the RNA molecules form dimers. At a lower RNA concentration used for functional in vitro assays $(\sim 6 \mu \mathrm{M})$, RNA molecules are predominantly monomeric, suggesting that dimerization is a crystallization artifact and not the functional state of the RNA (Supplemental Fig. S5A,B). Supporting this conclusion, the relative orientation of the two RNA molecules in a dimer is different in PLRV and SCNMV xrRNA crystals (Fig. 2D). The intermolecular base pairs involving $L 2 A_{P K}$ of one molecule and $\mathrm{J} 1 / 3_{\mathrm{PK}}$ of the other are nonetheless similarly mimicking the pseudoknot in both structures (Fig. 2C,D).

The interactions involving $\mathrm{J} 1 / 3_{\mathrm{CN}}$ (nucleotides $42-46$ in PLRV; Fig. 2A) immediately adjacent to $\mathrm{J} 1 / 3_{\mathrm{PK}}$ are also conserved in the PLRV and SCNMV structures (Fig. 4A$D)$. Examination of our sequence alignment reveals that these nucleotides are highly conserved, with $>97 \%$ con-
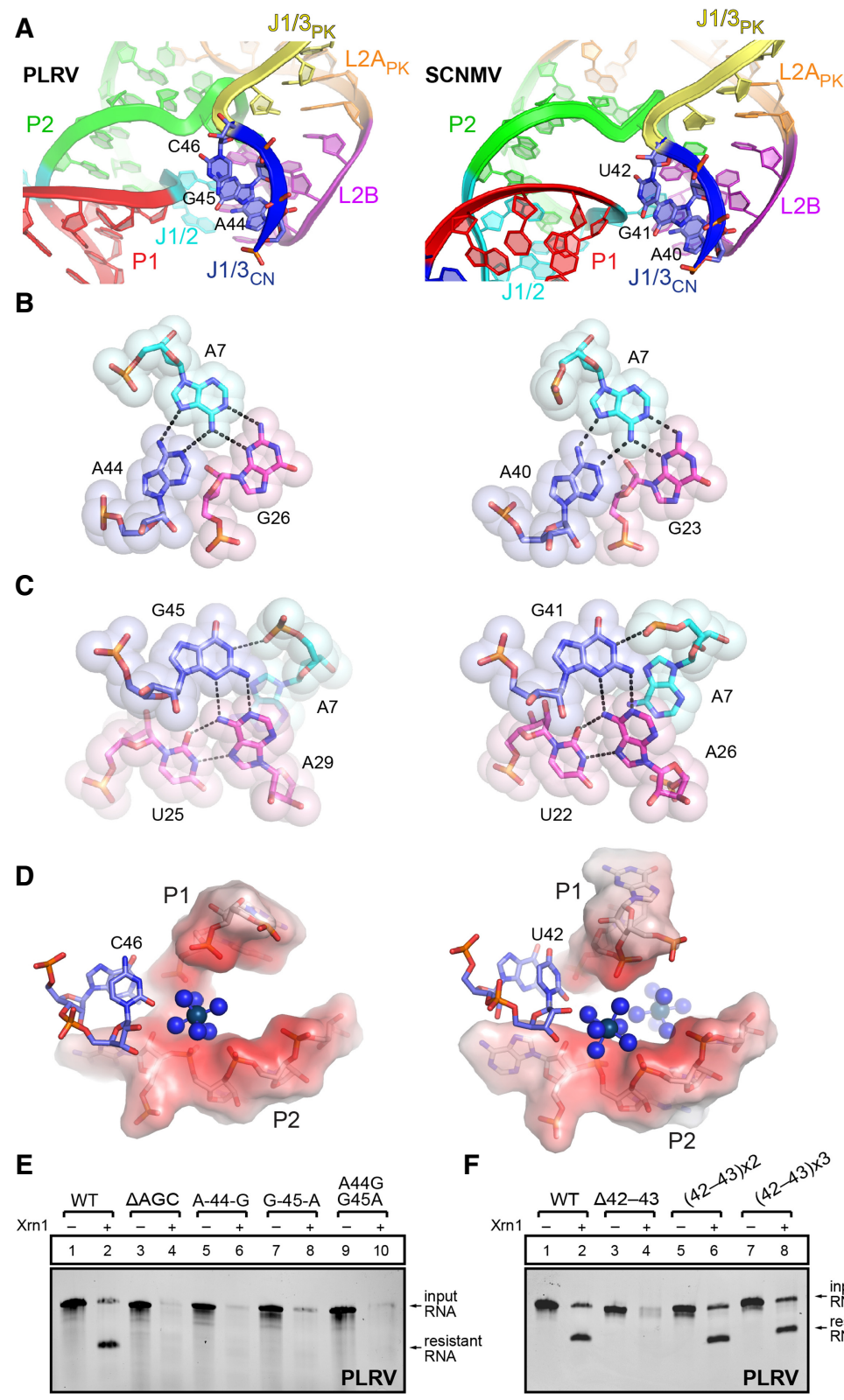

FIGURE 4. Conserved interactions involving $\mathrm{J} 1 / 3_{\mathrm{CN}}$ at the dimer interface. (A) Close-up on the region involving $\mathrm{J} 1 / 3_{\mathrm{CN}}$ (A44, G45, C46 in PLRV, and A40, G41, U42 in SCNMV) in both structures. For panels $A-D$, the PLRV structure on the left side is compared to the SCNMV on the right side. (B) Interaction network involving PLRV A44 (left) and SCNMV A40 (right). (C) Interaction network involving PLRV G45 (left) and SCNMV G41 (right). (D) C46 (PLRV)/U42 (SCNMV) are in the vicinity of a negatively charged region (in red) formed by the backbone atoms of $\mathrm{P} 1$ and $\mathrm{P} 2$. Iridium(III) hexammines are shown as blue spheres. $(E, F)$ In vitro $\mathrm{Xrn} 1$ degradation assays of PLRV xrRNA WT and J1/3 $\mathrm{CN}$ mutants.

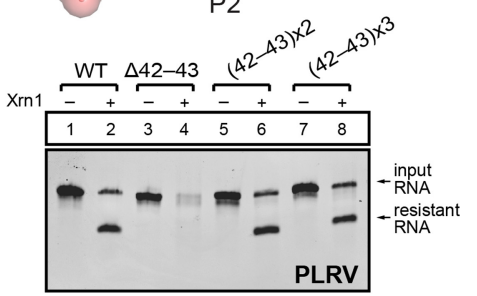

servation of A44 and G45 (A40 and G41 in SCNMV) and the conserved presence of a pyrimidine at position 46 (Fig. 1C). A44, G45, and U46 all stack together and help bridge the bent-over appearance of this hairpin. In particular, A44 interacts with the Hoogsteen side of A7 and is part of an interaction network involving $U 25$ and G26 from L2B. G45 stacks on A44 and bridges A7 to the 
conserved A29-U25 pair (Fig. 4C). Finally, C46/U42 points toward a negatively charged region formed by the backbone of $\mathrm{P} 1$ and $\mathrm{P} 2$, where iridium(III) hexammine complexes have been assigned in both PLRV and SCNMV (Fig. 4D). The absence of a purine at this position across XrRNA ${ }_{L T}$ sequences (Supplemental Fig. S1) could be justified by the steric clashes or the disturbance of potential physiological metal ion binding sites that a purine would cause.

To interrogate the importance of the conservation at $\mathrm{J} 1 / 3_{\mathrm{CN}}$, we generated mutants to disrupt the interaction involving nucleotides 44-46 (Supplemental Fig. S7) and tested them for exoribonuclease resistance. As expected, deletion of A44-C46 ( $\triangle A G C$ ) removes the ability to resist degradation by Xrn1 (Fig. 4E, lanes 3 and 4). But even purine to purine substitutions at positions 44 and/or 45 are sufficient to disrupt tertiary interactions, leading to complete degradation by $\mathrm{Xrn} 1$ (Fig. 4E, lanes 5-10). In conclusion, the conserved $5^{\prime}$-AGY- $3^{\prime}$ sequence in $\mathrm{J} 1 / 3_{\mathrm{CN}}$ is involved in tertiary interactions that are key for resistance against $\mathrm{Xrn} 1$ degradation. This suggests that the interactions occurring in trans within the dimer probably occur in cis to stabilize the active fold.

The equivalent of $\mathrm{J} 1 / 3_{\mathrm{CN}}$ in flavivirus xrRNA is part of the ring-like structure that wraps around the $5^{\prime}$ end (Chapman et al. 2014a; Steckelberg et al. 2018a), which suggests its length would be important for proper ring formation and hence resistance. Because $\mathrm{J} 1 / 3_{\mathrm{CN}}$ shows variation in length across $x r R N A_{L T}$ elements beyond the more frequent length of 6 nt (Supplemental Fig. S1), we aimed to test the effect on resistance of decreasing or increasing $\mathrm{J} 1 / 3_{\mathrm{CN}}$. As for $\mathrm{P} 2$ and $\mathrm{L} 2 \mathrm{~A}_{\mathrm{CN}}$ discussed above, $\mathrm{J} 1 / 3_{\mathrm{CN}}$ is on the shorter side for SCNMV (three nucleotides vs. five in PLRV; Fig. 1C). Shortening the $J 1 / 3_{\mathrm{CN}}$ in the PLRV $\operatorname{xrRNA}_{L T}$ as with the $\triangle \mathrm{AGC}$ mutant affected function (Fig. $4 \mathrm{E})$, although that effect is more likely due to the disruption of the associated interaction networks (Fig. 4A-D). Upon shortening $\mathrm{J} 1 / 3_{\mathrm{CN}}$ by two nucleotides upstream of the AGC stretch (deleting C42 and A43), resistance to Xrn1 was lost (Fig. 4F, lanes 3 and 4). This result suggested that this mutant may no longer be able to form a ring that would prevent it from being degraded. In contrast, extending J1/3 $\mathrm{CN}$ by $2 \mathrm{nt}[(42-43) \times 2]$ or $4 \mathrm{nt}[(42-43) \times 3]$ did not significantly reduce exoribonuclease resistance (Fig. 4F, lanes 5-8; Supplemental Fig. S7), indicating a tolerance for a somewhat longer $\mathrm{J} 1 / 3_{\mathrm{CN}}$, in line with the observed variation across $x r R N A_{L T}$ sequences.

\section{Conclusions}

The presence of xrRNAs in a growing list of viruses from several major superfamilies, combined with emerging structural information, continues to expand our insight into their diversity and evolution. The work presented here further shows that all xrRNA structures solved thus far have the potential to form a ring-like fold, but there are different ways to achieve it. In that sense, visualizing the closed conformation for an $\operatorname{xrRNA}_{L T}$-that is, with a ring encircling the $5^{\prime}$ end-remains a priority, as it would enable a thorough comparison across the currently known xrRNA active folds. The fact that xrRNAs from flaviviruses and dianthoviruses/poleroviruses are variations on the same theme suggests they may have evolved from a common precursor. Indeed, an emerging hypothesis in biology is that all viruses have a common ancestor (Wolf et al. 2018). Thus, continuing to expand our understanding of the relatedness of xrRNA folds will not only inform computational searches for additional versions, but will open the possibility to use the similarity of structured RNA elements with conserved functional roles as another criterion for virus relatedness, evolution, and taxonomy.

\section{MATERIALS AND METHODS}

\section{DNA templates and mutagenesis}

DNA templates for in vitro transcription were gBlocks ordered from IDT, cloned into pUC19 and verified by sequencing. RNA constructs for $\mathrm{Xrn} 1$ degradation assays contained the xrRNA sequence plus $34 \mathrm{nt}$ of the endogenous upstream sequence of the viral genome ("leader sequence") to allow loading of the exoribonucleases. Mutations were introduced using custom DNA primers (IDT) during PCR amplification of DNA templates (see "In vitro transcription" below). Nucleotide numbering throughout the manuscript is in reference to the crystallized RNA construct. Transcription with T7 RNA polymerase was started with two guanosine nucleotides for enhanced transcription efficiency.

\section{In vitro transcription}

DNA templates for in vitro transcription were amplified by PCR using custom DNA primers (IDT) and Phusion Hot Start polymerase (New England BioLabs). An amount of $2.5 \mathrm{~mL}$ transcription reactions were assembled using $1000 \mu \mathrm{L}$ PCR reactions as template $(\sim 0.2 \mu \mathrm{M}$ template DNA), $6 \mathrm{mM}$ each NTP, $60 \mathrm{mM}$ $\mathrm{MgCl}_{2}, 30 \mathrm{mM}$ Tris $\mathrm{pH}$ 8.0, $10 \mathrm{mM}$ DTT, $0.1 \%$ spermidine, $0.1 \%$ Triton X-100, T7 RNA polymerase, and $2 \mu \mathrm{L}$ RNasin RNase inhibitor (Promega) and incubated overnight at $37^{\circ} \mathrm{C}$. After inorganic pyrophosphates were precipitated by centrifugation, the reactions were ethanol precipitated and purified on a $7 \mathrm{M}$ urea $8 \%$ denaturing polyacrylamide gel. RNAs of the correct size were excised, eluted overnight at $4^{\circ} \mathrm{C}$ into $\sim 40 \mathrm{~mL}$ of diethylpyrocarbonate (DEPC)-treated milli-Q filtered water (Millipore) and concentrated and washed using Amicon Ultra spin concentrators (Millipore).

\section{Protein expression and purification}

The expression vector for Kluyveromyces lactis Xrn1 (Chang et al. 2011) (residues 1-1245) was a gift of Prof. Liang Tong at Columbia University and the expression vector for Bdellovibrio 
bacteriovorus RppH was a gift of Joel Belasco at NYU (Messing et al. 2009). All recombinant proteins were 6XHis-tagged, expressed in E. coli BL21 cells and purified using Ni-NTA resin (Thermo), followed by size exclusion with either a Superdex 75 or Superdex 200 column in an AKTA pure FPLC (GE Healthcare). The final product was stored in buffer containing $20 \mathrm{mM}$ Tris $\mathrm{pH} 7.3,300 \mathrm{mM} \mathrm{NaCl}, 1 \mathrm{mM}$ DTT, or $2 \mathrm{mM} \mathrm{BME}$, and $10 \%$ glycerol at $-80^{\circ} \mathrm{C}$. The purity of the recombinant proteins was verified by SDS-PAGE and Coomassie staining.

\section{5'-3' exoribonuclease degradation assay}

An amount of $4 \mu \mathrm{g}$ RNA was resuspended in $40 \mu \mathrm{L} 100 \mathrm{mM} \mathrm{NaCl}$, $10 \mathrm{mM} \mathrm{MgCl}_{2}, 50 \mathrm{mM}$ Tris $\mathrm{pH} 7.5$, and $1 \mathrm{mM} \mathrm{DTT}$ and refolded at $90^{\circ} \mathrm{C}$ for $3 \mathrm{~min}$ then $20^{\circ} \mathrm{C}$ for $5 \mathrm{~min}$. An amount of $3 \mu \mathrm{L}$ recombinant $\mathrm{RppH}(0.5 \mu \mathrm{g} / \mu \mathrm{L}$ stock) was added and the samples were split into two $20 \mu \mathrm{L}$ reactions ( \pm exoribonuclease). An amount of $1.5 \mu \mathrm{L}$ of the recombinant $\mathrm{Xrn} 1(0.8 \mu \mathrm{g} / \mu \mathrm{L}$ stock) was added where indicated. All reactions were incubated for $1.5 \mathrm{~h}$ at $30^{\circ} \mathrm{C}$ using a thermocycler. The degradation reactions were resolved on a $7 \mathrm{M}$ urea $10 \%$ denaturing polyacrylamide gel and stained with ethidium bromide. Where indicated, guanidine hydrochloride $(1,10$, or $100 \mathrm{mM}$ final concentration) was added to the RNA folding buffer before heat-denaturing the RNA at $90^{\circ} \mathrm{C}$.

\section{${ }^{32} \mathrm{P}-5^{\prime}$ end-labeling of RNA}

RNA was dephosphorylated using rApid Alkaline Phosphatase (Millipore) to convert 5'-triphosphate to $5^{\prime}$-monophosphate ends. To this end, 100 pmol RNA was incubated in a $20 \mu \mathrm{L}$ reaction containing $2 \mu \mathrm{L}$ rApid Alkaline phosphatase buffer, $2 \mu \mathrm{L}$ rApid Alkaline phosphatase, $1 \mu \mathrm{L}$ RNasin Ribonuclease Inhibitor (SigmaAldrich), and incubated for $30 \mathrm{~min}$ at $37^{\circ} \mathrm{C}$. The enzyme was heatinactivated for 2 min at $75^{\circ} \mathrm{C}$. The RNA was subsequently $5^{\prime}$ endlabeled with [ $\gamma 32-P]$ ATP (PerkinElmer) using T4 polynucleotide kinase (PNK) (New England Biolabs). This was done by adding $1 \mu \mathrm{L}$ $100 \mathrm{mM} \mathrm{MgCl}_{2}, 4 \mu \mathrm{L}$ PNK Buffer, $2 \mu \mathrm{L}$ of $5 \mathrm{mCi}[\gamma 32-\mathrm{P}]$ ATP, and $2 \mu \mathrm{L}$ of PNK in a total volume of $40 \mu \mathrm{L}$. The reaction mix was incubated for $30 \mathrm{~min}$ at $37^{\circ} \mathrm{C}$ and purified using Micro Bio P-30 spin columns (Bio-Rad).

\section{Electrophoretic mobility shift assay}

A 1:1 serial dilution of unlabeled RNA ranging from $600 \mu \mathrm{M}$ to $9.4 \mu \mathrm{M}$ was diluted in $6 \mu \mathrm{L} 1 \times \mathrm{TH}$-loading buffer $(66 \mathrm{mM}$ Tris$\mathrm{HCl}, 34 \mathrm{mM}$ HEPES, $5 \mathrm{mM} \mathrm{MgCl}$, 10\% glycerol, xylene cyanol, bromophenol blue) supplemented with trace amounts of ${ }^{32} \mathrm{P}$ labeled RNA, heated for $3 \mathrm{~min}$ at $85^{\circ} \mathrm{C}$, followed by $5 \mathrm{~min}$ at $20^{\circ} \mathrm{C}$, cooled to $4^{\circ} \mathrm{C}$, and loaded on an $8 \% 1 \times \mathrm{TH}$-polyacrylamide native gel (8\% polyacrylamide/bis solution 29:1, $66 \mathrm{mM}$ Tris- $\mathrm{HCl}$, $34 \mathrm{mM}$ HEPES, $5 \mathrm{mM} \mathrm{MgCl} 2$ ). The gels were run at $70 \mathrm{~V}$ at $4^{\circ} \mathrm{C}$ in $1 \times$ TH buffer ( $66 \mathrm{mM}$ Tris-HCl, $34 \mathrm{mM}$ HEPES, $5 \mathrm{mM} \mathrm{MgCl}_{2}$ ) until the bromophenol blue dye front reached the lower quarter of the gel $(\sim 3 \mathrm{~h})$, then imaged using a phosphor screen and Typhoon 9400 scanner (GE Life Sciences). Where indicated, 100 mM guanidine hydrochloride was added to the $\mathrm{TH}$-loading buffer, the $\mathrm{TH}$-polyacrylamide gel, and the TH-running buffer.

\section{RNA crystallization and diffraction data collection}

RNA for crystallization was prepared as described above. The sequence used for in vitro transcription of the RNA that was used for structure determination was

\section{5'-ggAACTAGCtcAGCATACACGAGTTGCAAGCATgGGAAGTT CAAGCCTCGTGGGCGGCAT \\ GGTCCCAGCCTCCTCGCTGGCGCCGCCTGGGCAACATGCTT CGGCATGGCGAATGGGACC-3'}

where the underlined sequence belongs to a hepatitis delta ribozyme that was used to generate homogenous $3^{\prime}$ ends. Lower case letters represent sequences altered to facilitate transcription (two additional $G$ s on the $5^{\prime}$ end) and the G.U phasing module. Ribozyme cleavage was induced at the end of the transcription reaction by adding $\mathrm{MgCl}_{2}$ (final conc. $120 \mathrm{mM}$ ) and incubating for $10 \mathrm{~min}$ at $65^{\circ} \mathrm{C}$. Ribozyme-cleaved RNA was purified on a $7 \mathrm{M}$ urea $8 \%$ polyacrylamide gel as described above. $5 \mathrm{mg} / \mathrm{mL}$ RNA was refolded at $65^{\circ} \mathrm{C}$ for $3 \mathrm{~min}$ in a buffer containing $30 \mathrm{mM}$ HEPES pH 7.5, $20 \mathrm{mM} \mathrm{MgCl}_{2}$, and $100 \mathrm{mM} \mathrm{KCl}$. Crystal Screens I and II, Natrix I and II, and the Nucleic Acid Mini Screen (all from Hampton Research) were used to perform initial screens at $20^{\circ} \mathrm{C}$ with sitting-drop vapor diffusion crystallization. Initial hits were optimized using custom screens and the Hampton Research Additive Screen.

The RNAs used for the final structural determination were crystallized in drops of $1 \mu \mathrm{L}$ RNA solution $(5 \mathrm{mg} / \mathrm{mL}$ RNA in $30 \mathrm{mM}$ HEPES pH 7.5, 20 mM MgCl $2,100 \mathrm{mM} \mathrm{KCl})+1 \mu \mathrm{L}$ crystallization solution (10\% 2-methyl-2,4-pentanediol, $40 \mathrm{mM}$ Sodium Cacodylate $\mathrm{pH}$ 6.0, $12 \mathrm{mM}$ Spermine, $150 \mathrm{mM} \mathrm{KCl}, 100 \mathrm{mM}$ Guanidinium $\mathrm{HCl}$ ) over a reservoir of $500 \mu \mathrm{L}$ of $30 \%$ 2-methyl2,4-pentanediol and $100 \mathrm{mM}$ Guanidinium $\mathrm{HCl}$ at $20^{\circ} \mathrm{C}$ with sitting-drop vapor diffusion crystallization. Crystals appeared overnight and grew over the course of several days to a final size of 200-300 $\mu \mathrm{m}$ in their longest dimension. Crystals were buffer-exchanged into freezing solution (30\% 2-methyl-2,4-pentanediol, 40 mM Sodium Cacodylate pH 6.0, 12 mM Spermine, 150 mM $\mathrm{KCl}, 100 \mathrm{mM}$ Guanidinium $\mathrm{HCl}$ ), with or without $5 \mathrm{mM}$ iridium (III) hexammine to obtain derived crystals for experimental SAD phasing, and then flash-frozen in liquid nitrogen for X-ray diffraction. Diffraction data were collected at Advanced Light Source Beamline 4.2.2 using "shutterless" collection at the Iridium L-I edge $(0.9234 \AA)$ at $100 \mathrm{~K}$ and using a CMOS RDI 8M detector. Two native and three derivative $180^{\circ}$ data sets were collected with $0.2^{\circ}$ oscillation images. Data were indexed, integrated, and scaled using XDS (Kabsch 2010). Three data sets collected on the same crystal were used for SAD phasing.

\section{Structure determination and refinement}

Ten iridium(III) hexammine sites were identified and used in SAD phasing within the AutoSol function of Phenix v. 1.13_2998 on Mac OS (overall FOM $=0.48$ ) (Liebschner et al. 2019). The map was used to manually build an initial model, which was improved through iterative rounds of model building and refinement (simulated annealing, Rigid-body, B-factor refinement) using Coot (Emsley et al. 2010) and Phenix (Liebschner et al. 2019). The final model contains two RNA molecules in the asymmetric unit and all $51 \mathrm{nt}$ are resolved. 
Peaks in the anomalous map disappearing around 5 sigma were assigned to cacodylate molecules ( $f^{\prime \prime}($ As) $\sim 3$ electrons at $0.9234 \AA$ ) , also taking geometry into account. The model was refined in Phenix v. 1.17.1_3660 on Mac OS, using the graphic user interface, down to $R / R_{\text {free }}$ of $0.25 / 0.28$. The GTP introduced upon transcription at the $5^{\prime}$ end and the $2^{\prime}, 3^{\prime}$ cyclic UMP introduced during ribozyme cleavage at the $3^{\prime}$ end were linked to the RNA chain using JLigand in CCP4i v. 7.0 (Winn et al. 2011; Lebedev et al. 2012). Map calculation in Refmac v. 5.8.0257 within CCP4i (Winn et al. 2011) led to a higher-quality map with clear density peaks in both $2\left|F_{o}\right|-\left|F_{c}\right|$ and $\left|F_{o}\right|-\left|F_{c}\right|$ for the guanidinium ligands. The structure was further refined in Refmac to $R / R_{\text {free }}$ $0.23 / 0.27$. The deposited structure $\left(R / R_{\text {free }}\right.$ of $\left.0.225 / 0.254\right)$ was obtained from phenix.refine using default parameters for TLS refinement, occupancy refinement, optimization of X-ray/stereochemistry and X-ray/B-factor weights, and ligand CIF files that were adjusted from the Phenix default geometry parameters and partial charges (available as Supplemental Material). In particular, C-N distances within guanidinium were changed from 1.252 $\AA$ (double) and $1.452 \AA$ (single) to $1.37 \AA$ (each $\mathrm{C}-\mathrm{N}$ bond is delocalized) (Adams and Small 1974; Sawinski et al. 2013). Charges were distributed as follows: +0.3 on the $C$ atom and +0.233 on each $\mathrm{N}$ atom. For iridium(III) hexammine, the Ir- $\mathrm{N}$ distance was changed from $2.035 \AA$ to $2.1 \AA$ (Gorol et al. 2000), a +1 charge was placed at each amine, and a -3 charge was placed at the Ir atom. Together, these changes led to better interpretation of the electron density, as reflected by drops in B-factors and overall $R_{\text {free. }}$. Finally, the geometry of the PLRV xrRNA $A_{L T}$ structure was validated using the MolProbity web server (Williams et al. 2018).

\section{DATA DEPOSITION}

The coordinates have been deposited in the Protein Data Bank with accession code 7JJU.

\section{SUPPLEMENTAL MATERIAL}

Supplemental material is available for this article.

\section{ACKNOWLEDGMENTS}

We wish to thank Pascal Auffinger for helpful suggestions during refinement, and Jack Harrowfield for discussions about iridium(III) hexammine. Support was provided by the National Institutes of Health (grants R35GM118070 and R01Al133348 to J.S.K.) and the Deutsche Forschungsgemeinschaft Fellowship STE2509/201 to A.-L.S.). Beamline 4.2.2 at the Advanced Light Source is partially funded by the National Institutes of Health (P30GM124169-01) and operated under contract with the U.S. Department of Energy (DE-AC02-05CH11231). The University of Colorado Anschutz Medical Campus X-ray crystallography facility is supported by the National Institutes of Health (grants P30CA046934 and S10OD012033).

Received April 30, 2020; accepted August 19, 2020.

\section{REFERENCES}

Adams JM, Small RWH. 1974. The crystal structure of guanidinium carbonate. Acta Cryst B30: 2191-2193.

Akiyama BM, Laurence HM, Massey AR, Costantino DA, Xie X, Yang Y, Shi P-Y, Nix JC, Beckham JD, Kieft JS. 2016. Zika virus produces noncoding RNAs using a multi-pseudoknot structure that confounds a cellular exonuclease. Science 354: 1148-1152.

Batey RT, Kieft JS. 2016. Soaking hexammine cations into RNA crystals to obtain derivatives for phasing diffraction data. Methods Mol Biol 1320: 219-232.

Breaker RR, Atilho RM, Malkowski SN, Nelson JW, Sherlock ME. 2017. The biology of free guanidine as revealed by riboswitches. Biochemistry 56: 345-347.

Chang JH, Xiang S, Xiang K, Manley JL, Tong L. 2011. Structural and biochemical studies of the $5^{\prime} \rightarrow 3^{\prime}$ exoribonuclease Xrn1. Nat Struct Mol Biol 18: 270-276.

Chapman EG, Costantino DA, Rabe JL, Moon SL, Wilusz J, Nix JC, Kieft JS. 2014a. The structural basis of pathogenic subgenomic flavivirus RNA (sfRNA) production. Science 344: 307-310.

Chapman EG, Moon SL, Wilusz J, Kieft JS. 2014b. RNA structures that resist degradation by $\mathrm{Xrn} 1$ produce a pathogenic dengue virus RNA. elife 3: e01892.

Di Tondo P, Pritchard RG. 2012. Pentaguanidinium monohydrogendiphosphopentamolybdate(VI) 2.5-hydrate, (CH6N3)5[HP2Mo5O23]. $2.5 \mathrm{H} 2 \mathrm{O}$ : hydrogen bonding and pi-stacking in guanidinium cations. Acta Crystallogr C 68: i50-i52.

Emsley P, Lohkamp B, Scott WG, Cowtan K. 2010. Features and development of Coot. Acta Crystallogr D Biol Crystallogr 66: 486-501.

Gorol M, Mösch-Zanetti NC, Noltemeyer M, Roesky HW. 2000. Water-soluble and halogen-free hexaammine complexes of metal ions of group 9-synthesis, crystal structures, and vibrational spectra. Zeitschrift für anorganische Chemie 626: 23182324.

Iwakawa H-O, Mizumoto H, Nagano H, Imoto Y, Takigawa K, Sarawaneeyaruk S, Kaido M, Mise K, Okuno T. 2008. A viral noncoding RNA generated by cis-element-mediated protection against $5^{\prime} \rightarrow 3^{\prime}$ RNA decay represses both cap-independent and cap-dependent translation. J Virol 82: 10162-10174.

Jones RA, Steckelberg AL, Vicens Q, Szucs MJ, Akiyama BM, Kieft JS. 2020. Different tertiary interactions create the same important 3-D features in a distinct flavivirus xrRNA. RNA doi:10.1261/rna .077065 .120

Kabsch W. 2010. XDS. Acta Crystallogr D Biol Crystallogr 66: 125132.

Keel AY, Rambo RP, Batey RT, Kieft JS. 2007. A general strategy to solve the phase problem in RNA crystallography. Structure 15: 761-772.

Krueger EN, Beckett RJ, Gray SM, Miller WA. 2013. The complete nucleotide sequence of the genome of Barley yellow dwarf virusRMV reveals it to be a new Polerovirus distantly related to other yellow dwarf viruses. Front Microbiol 4: 205.

Lebedev AA, Young $P$, Isupov MN, Moroz OV, Vagin AA, Murshudov GN. 2012. JLigand: a graphical tool for the CCP4 template-restraint library. Acta Crystallogr D Biol Crystallogr 68: 431440.

Leontis NB, Westhof E. 2001. Geometric nomenclature and classification of RNA base pairs. RNA 7: 499-512.

Liebschner D, Afonine PV, Baker ML, Bunkóczi G, Chen VB, Croll TI, Hintze B, Hung LW, Jain S, McCoy AJ, et al. 2019. Macromolecular structure determination using X-rays, neutrons and electrons: recent developments in Phenix. Acta Crystallogr D Struct Biol 75: 861-877.

MacFadden A, O'Donoghue Z, Silva PAGC, Chapman EG, Olsthoorn RC, Sterken MG, Pijlman GP, Bredenbeek PJ, 
Kieft JS. 2018. Mechanism and structural diversity of exoribonuclease-resistant RNA structures in flaviviral RNAs. Nat Commun 9: 119.

Mahuku G, Lockhart BE, Wanjala B, Jones MW, Kimunye JN, Stewart LR, Cassone BJ, Sevgan S, Nyasani JO, Kusia E, et al. 2015. Maize lethal necrosis (MLN), an emerging threat to maizebased food security in sub-saharan Africa. Phytopathology 105: 956-965.

Messing SAJ, Gabelli SB, Liu Q, Celesnik H, Belasco JG, Piñeiro SA, Amzel LM. 2009. Structure and biological function of the RNA pyrophosphohydrolase BdRppH from Bdellovibrio bacteriovorus. Structure 17: 472-481.

Nelson JW, Atilho RM, Sherlock ME, Stockbridge RB, Breaker RR. 2017. Metabolism of free guanidine in bacteria is regulated by a widespread riboswitch class. Mol Cell 65: 220-230.

Perry KL, Kolb FL, Sammons B, Lawson C, Cisar G, Ohm H. 2000. Yield effects of Barley yellow dwarf virus in soft red winter wheat. Phytopathology 90: 1043-1048.

Pijlman GP, Funk A, Kondratieva N, Leung J, Torres S, van der Aa L, Liu WJ, Palmenberg AC, Shi P-Y, Hall RA, et al. 2008. A highly structured, nuclease-resistant, noncoding RNA produced by flaviviruses is required for pathogenicity. Cell Host Microbe 4: 579591.

Reiss CW, Strobel SA. 2017. Structural basis for ligand binding to the guanidine-II riboswitch. RNA 23: 1338-1343.

Sawinski PK, Meven M, Englert U, Dronskowski R. 2013. Single-crystal neutron diffraction study on guanidine, CN3H5. Crystal Growth Design 13: 1730-1735.
Slonchak A, Khromykh AA. 2018. Subgenomic flaviviral RNAs: what do we know after the first decade of research. Antiviral Res 159: 13-25.

Steckelberg A-L, Akiyama BM, Costantino DA, Sit TL, Nix JC, Kieft JS. 2018a. A folded viral noncoding RNA blocks host cell exoribonucleases through a conformationally dynamic RNA structure. Proc Natl Acad Sci 115: 6404-6409.

Steckelberg A-L, Vicens Q, Kieft JS. 2018b. Exoribonuclease-resistant RNAs exist within both coding and noncoding subgenomic RNAs. MBio 9: e02461-18.

Vazdar M, Heyda J, Mason PE, Tesei G, Allolio C, Lund M, Jungwirth P. 2018. Arginine "Magic": guanidinium like-charge ion pairing from aqueous salts to cell penetrating peptides. Acc Chem Res 51: 1455-1464.

Wale SJ, Platt HW, Cattlin ND. 2008. Virus and phytoplasma diseases. In Diseases, pests and disorders of potatoes: a color handbook, pp. 75-92. Manson Publishing, London.

Williams CJ, Headd JJ, Moriarty NW, Prisant MG, Videau LL, Deis LN, Verma V, Keedy DA, Hintze BJ, Chen VB, et al. 2018. MolProbity: more and better reference data for improved all-atom structure validation. Protein Sci 27: 293-315.

Winn MD, Ballard CC, Cowtan KD, Dodson EJ, Emsley P, Evans PR, Keegan RM, Krissinel EB, Leslie AGW, McCoy A, et al. 2011. Overview of the CCP4 suite and current developments. Acta Crystallogr D Biol Crystallogr 67: 235-242.

Wolf YI, Kazlauskas D, Iranzo J, Lucía-Sanz A, Kuhn JH, Krupovic M, Dolja WV, Koonin EV. 2018. Origins and evolution of the global RNA virome. MBio 9: e02329-18. 

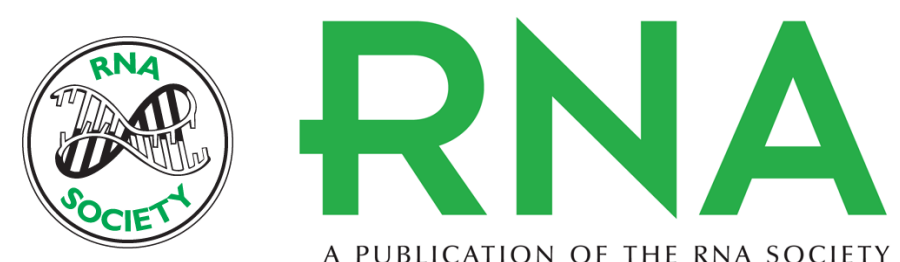

A PUBLICATION OF THE RNA SOCIETY

\section{The crystal structure of a Polerovirus exoribonuclease-resistant RNA shows how diverse sequences are integrated into a conserved fold}

Anna-Lena Steckelberg, Quentin Vicens, David A. Costantino, et al.

RNA 2020 26: 1767-1776 originally published online August 26, 2020

Access the most recent version at doi:10.1261/rna.076224.120

\section{Supplemental http://rnajournal.cshlp.org/content/suppl/2020/08/26/rna.076224.120.DC1 \\ Material}

References This article cites 33 articles, 8 of which can be accessed free at: http://rnajournal.cshlp.org/content/26/12/1767.full.html\#ref-list-1

Creative This article is distributed exclusively by the RNA Society for the first 12 months after the Commons

License full-issue publication date (see http://rnajournal.cshlp.org/site/misc/terms.xhtml). After 12 months, it is available under a Creative Commons License (Attribution-NonCommercial 4.0 International), as described at http://creativecommons.org/licenses/by-nc/4.0/.

Email Alerting
Service

Receive free email alerts when new articles cite this article - sign up in the box at the top right corner of the article or click here.

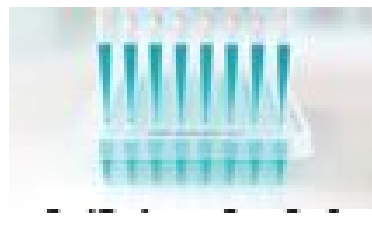

Providing Precise Solutions for your research.

To subscribe to $R N A$ go to:

http://rnajournal.cshlp.org/subscriptions 